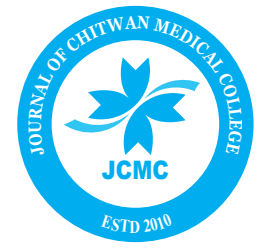

Journal of Chitwan Medical College 2019;9(27):74-77

Available online at: www.jcmc.cmc.edu.np

\section{GISE BEPORT}

FETAL HEPATIC HEMANGIOMA: A CASE REPORT

\author{
Manindra Shrestha ${ }^{1,}{ }^{,}$, Nishma Bajracharya ${ }^{2}$, Ashish Parajuli ${ }^{1}$, Amritee Gorkhali ${ }^{1}$, Amir Shrestha ${ }^{1}$, Ganesh Dangal ${ }^{2}$ \\ ${ }^{1}$ Department of Radiology, Kathmandu Model Hospital, Exhibition Road, Kathmandu, Nepal. \\ ${ }^{2}$ Department of Obstetrics and Gynecology, Kathmandu Model Hospital, Exhibition Road, Kathmandu, Nepal..
}

Received: 12 Feb, 2019

Accepted: 23 March, 2019

Published: 31 March, 2019

Key words: Arteriovenous shunting; Fetal Hepatic Hemangioma; Ultrasonography.

*Correspondence to: Manindra Shrestha, Department of Radiology, Kathmandu Model Hospital, Exhibition Road, Kathmandu, Nepal. Email: manamshrestha@gmail.com

\section{Citation}

Shrestha M, Bajracharya N, Parajuli A, Gorkhali A, Shrestha A, Dangal G. Fetal hepatic hemangioma: a case report. Journal of Chitwan Medical College. 2019; 9 (27): 74-77.

\begin{abstract}
Fetal liver tumors represent approximately $5 \%$ of all congenital tumors and hemangiomas account for approximately $15 \%$ of all fetal liver tumors. Hemangioma and hemangioendothelioma are benign vascular tumors but they are associated with a significant morbidity and mortality due to high output congestive heart failure and arteriovenous shunting. Ultrasound features of hepatic hemangioma can overlap with arteriovenous malformation. This is a case report of fetal hepatic hemangioma with aretriovenous shunting detected on routine anomaly scan at 20 weeks of gestation in 22 years old Nepalese women who was asymptomatic. Prenatally, a $3.2 \mathrm{~cm} \times 2.8 \mathrm{~cm}$ multicystic mass was seen in the left hypochondrium of the fetal abdomen displacing the stomach. On color Doppler examination, the mass showed internal color flow and multiple tortuous dilated vessels noted around the lesion. After counseling regarding the lesion to the mother and her family; decision to abort the fetus was made. Autopsy was done for the final diagnosis of the lesion, which showed vascular mass in the left lobe of liver insinuating between stomach and spleen.
\end{abstract}

\section{INTRODUCTION}

Hepatic tumors are rare in a perinatal period. They represent about $5 \%$ of all neoplasms. Various types of hepatic tumors include hemangioma, hemangioendothelioma, mesenchymal hamartoma and hepatoblastoma. ${ }^{1}$ Nearly, $15 \%$ of all fetal tumors are hemangiomas, which usually appear on the face, neck and limbs. Hemangioma and hemangioendothelioma are benign vascular tumors but they are associated with a significant morbidity and mortality due to high output congestive heart failure and arteriovenous shunting. ${ }^{2}$ They are characterized as either rapidly involuting or noninvoluting based on their clinical progression. Fetal hepatic hemangiomas are rarely reported; hence, their exact incidence rate remains undetermined. ${ }^{3}$ Congenital hepatic hemangiomas, may reach maximum size at birth and, in some cases, gradually disappear. Small hepatic hemangiomas are often asymptomatic and seldom require treatment whereas, huge hepatic hemangiomas (Diameter $>4$ 
$\mathrm{cm})$ are less frequently reported and can cause serious complications. ${ }^{4}$ Neonatal mortality rates can reach $30-100 \%$ if untreated. ${ }^{1}$ Hence, studies on the prenatal diagnosis of hepatic hemangiomas are necessary for thoroughly monitoring fetuses for tumor progression and related complications and help obstetricians decide on timely delivery and appropriate neonatal treatments. This is case report of a 22-year-old Primigravida who presented with fetal congenital hepatic hemangioma on routine anomaly scan with arteriovenous shunting.

\section{CASE REPORT}

A 22 years-old, healthy, Primigravida Nepalese woman presented for an obstetric anomaly ultrasound scan at 20 weeks of gestational age (GA) to our radiology department. Until then she had only one scan at 8 weeks of pregnancy. A $3.2 \mathrm{~cm} \times 2.8 \mathrm{~cm}$ multicystic mass was seen in the left hypochondrium of the fetal abdomen displacing the stomach (Figure 1).

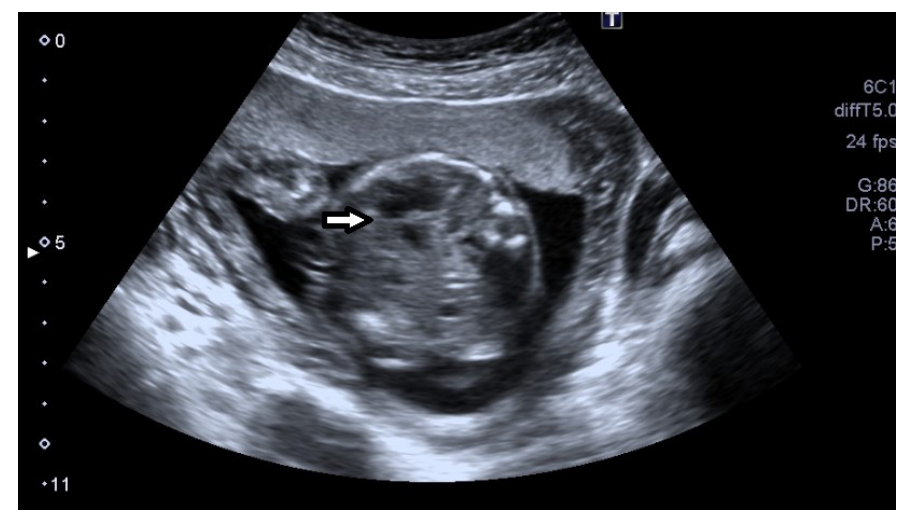

Figure 1. Lobulated multicystic mass of approx. ,3.2 $x 2.8 \mathrm{~cm}$ size (arrow) in left hypochondrium of fetal abdomen displacing the stomach.

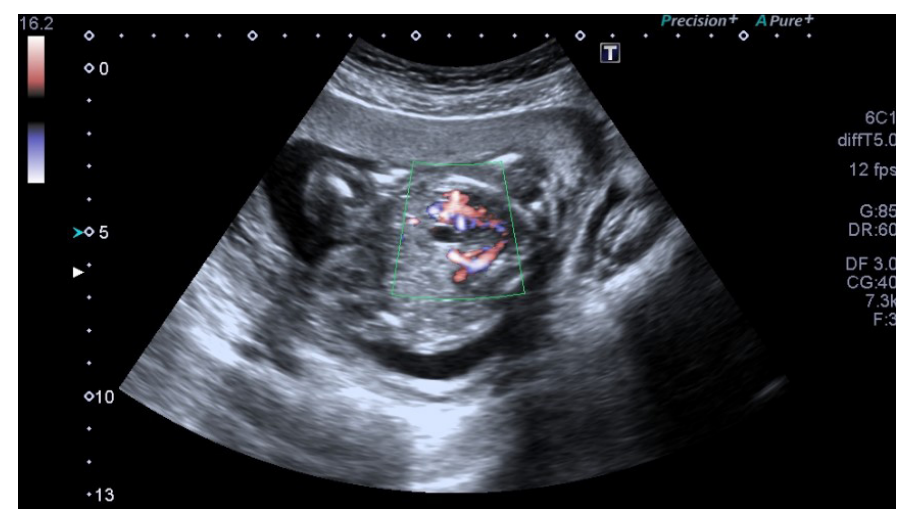

Figure 2. Color flow in the mass with tortuous vessels.

On color Doppler examination, the mass showed internal color flow and multiple tortuous dilated vessels noted around the lesion (Figure 2). Most of the vessels shows venous spectral Doppler wave form and few peripheral vessels shows arterial pattern. The initial diagnosis was made of vascular malformation of abdomen. Except this lesion, there were no other structural abnormalities noted in the fetus. Fetal cardiac size and chambers were normal. There was no history of any medical illness noted in the mother. After counseling regarding the lesion to the mother and her family; decision to abort the fetus was made. Taking the medicolegal written consent from patient, the fetus was aborted at 21 weeks of GA. Autopsy was done for the final diagnosis of the lesion, which showed vascular mass in the left lobe of liver insinuating between stomach and spleen (Figure 3).

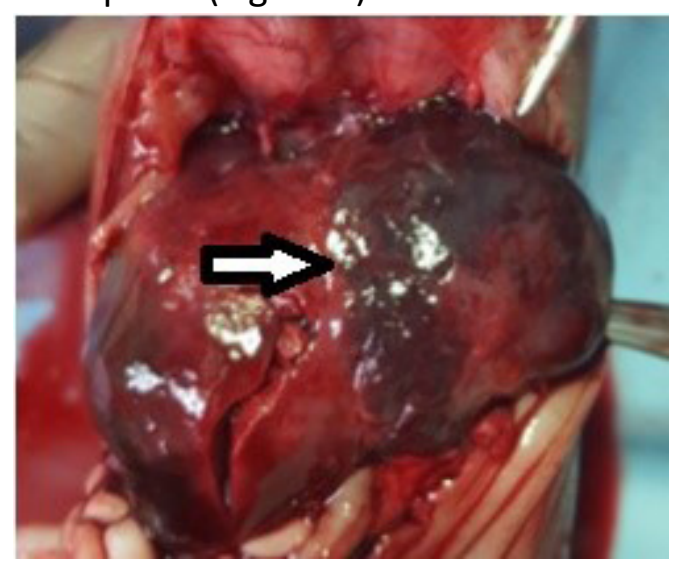

Figure 3. Bluish vascular mass approx $3 \times 2.5 \mathrm{~cm}$ in left lobe of liver (arrow).

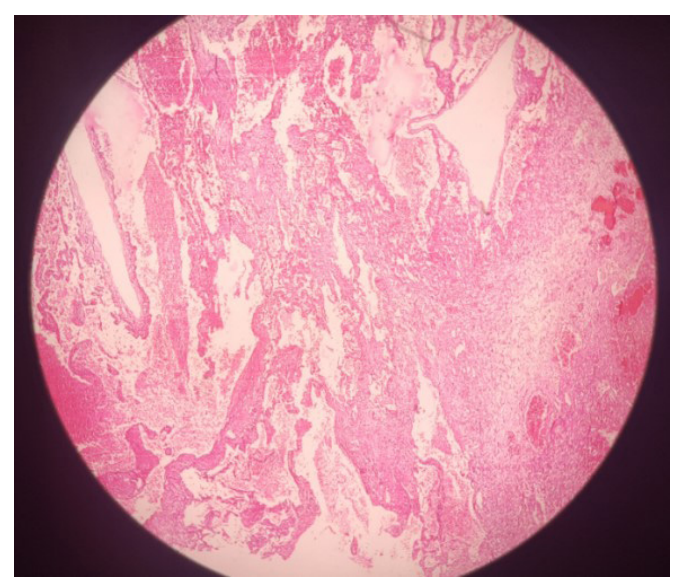

Figure 4. HE staining showing dilated vascular channels lined by a single layer of endothelial cells.

No other morphological abnormalities noted in the fetus. The histopathology report showed the cavernous hemangioma of the liver (Figure 4). 


\section{DISCUSSION}

The imaging findings of fetal hepatic tumors may widely overlap, the early detection and prenatal follow up of these tumors are very important for fetal, maternal, and postnatal care. ${ }^{5}$ In our case, such tumor presented as multicystic highly vascular mass in the left hypochondrium, diagnosed at routine congenital anomaly scan. The two categories of congenital vascular anomalies of the liver are proliferating tumors, such as hemangiomas, and developmental vascular disorders, such as arteriovenous malformations(AVMs). ${ }^{2}$ As proposed by Mulliken and Glowacki and according to the International Society for the Study of Vascular Anomalies (ISSVA), hemangiomas that may affect the liver in children can be classified as: infantile hemangiomas and congenital hemangiomas. Congenital hemangiomas may be Rapidly Involuting congenital hemangioma ( $\mathrm{RICH})$ or Non involuting congenital hemangioma $(\mathrm{NICH}) .{ }^{6}$ While small hemangiomas can be asymptomatic lesions incidentally discovered during imaging of the abdomen, larger tumors may act as arteriovenous shunts resulting in high-output congestive heart failure and hepatic dysfunction that may lead to fetal non-immune hydrops. The reported neonatal mortality rate of fetuses diagnosed with liver lesions is $30-100 \% .^{7}$ This prognosis lead to termination of pregnancy as a possible treatment option prenatally, which was seen in our case too. Since biopsy may result in massive haemorrhage, diagnosis is most often based on characteristic radiological findings of usually well-defined mixed, solid lesions with hypervascularization and fine granular calcifications. Although hemangiomas have been reported hyperechoic or isoechoic, our case showed a multi-septated complex cystic structure with low resistance flow. Associated, peripheral dilated vessels were also noted with low resistance flow. Sonographically, AVMs characteristically appear as echopenic dilated vascular channels replacing liver parenchyma with high-flow Doppler characteristics that lack arterial pulsation. Similar appearance was detected in our case showing anechoic area with peripheral tortuous vessels which is an indication of ateriovenous shunting. Hepatic hemangiomas can occur in either or both lobes of the liver but incidence is more in right lobe than left lobe. The lesions can be solitary or multifocal and vary in size. ${ }^{8}$ In case report, the fetus had a left-lobe lesion. When the lesion is in left lobe, the lesion can displace the adjacent organ i.e stomach and spleen such as in our case. Cases of fetal multiple hepatic hemangiomas have been reported rarely. ${ }^{6}$

Fatal complications of huge fetal hepatic hemangiomas include congestive heart failure, consumptive coagulation dysfunction (KasabachMerritt syndrome), and liver rupture. ${ }^{9}$ Hence, evaluation of intrauterine conditions via serial ultrasound examinations is important in making pregnancy-related decisions, including active management with in-utero treatment, expectant management, or expediting delivery. In resource poor areas like ours, termination of pregnancy is one of the options for the patient, and so it was in our case.

\section{CONCLUSION}

Congenital hepatic hemangioma in fetus is not uncommon, but detection of the lesion is crucial in management, as there is overlap in sonographic findings particularly with AVM. And associated, life threatening conditions of hemangioma resulting in increased mortality should always to be considered. Detailed anomaly scan plays an important role to detect any vascular anomaly in fetus. Serial imaging should be performed to evaluate the characteristics of lesion and to avoid any related complications.

\section{REFERENCES}

1. Isaacs $\mathrm{H}$, Jr. Fetal and neonatal hepatic tumors. J Pediatr Surg. 2007 Nov;42(11):1797-803. https:// doi.org/10.1016/j.jpedsurg.2007.07.047

2. Botha T, Rasmussen O, Carlan SJ, Greenbaum L. Congenital hepatic arteriovenous malformation: sonographic findings and clinical implications. Journal of Diagnostic Medical Sonography. 2004 Jun;20(3):177-81. https://doi. org/10.1177/8756479304263512

3. Jiao-ling $L$, Xiu-ping $G$, Kun-shan $C$, Qiu-ming $H$, Xiao-fen L, Bo-yang Y, Qian F. Huge fetal hepatic Hemangioma: prenatal diagnosis on ultrasound and prognosis. BMC Pregnancy and Childbirth. 2018 Dec;18(1):2. https://doi.org/10.1186/ s12884-017-1635-7

4. Chuileannain FN, Rowlands S, Sampson 
A. Ultrasonographic appearances of fetal hepatic hemangioma. J Ultrasound Med. 1999;18(5):379-81.https://dx.doi.org/10.7863/

5. Cho JY, Lee YH. Fetal tumors: prenatal ultrasonographic findings and clinical characteristics. Ultrasonography. 2014 Oct;33(4):240.https://doi.org/10.14366/ usg.14019

6. Mulliken JB, Glowacki J. Hemangiomas and vascular malformations in infants and children: a classification based on endothelial characteristics. Plast Reconstr Sur. 1982;69:412. https://www. ncbi.nlm.nih.gov/pubmed/7063565

7. Franchi-Abella $S$, Gorincour G, Avni F, Guibaud L, Chevret L, Pariente D. SFIPPGRRIF (Société Francophone d'Imagerie Pédiatrique et Périnatale-Groupe de Recherche Radiopédiatrique en Imagerie Foetale). Hepatic haemangioma-prenatal imaging findings, complications and perinatal outcome in a case series. Pediatr Radiol. 2012;42(3):298-307. https://dx.doi.org/10.1007/s00247-011-2214-0

8. Lutgendorf $M A$, Magann $E F$, Yousef $M$, Hill JB, Foster DT. Hepatic epithelial hemangioendothelioma in pregnancy. Gynecol Obstet Investig. 2009;67(4):238-40.https:// dx.doi.org/10.1159/000209216

9. Gembruch $U$, Baschat AA, Gloeckner-Hoffmann K, Gortner L, Germer U. Prenatal diagnosis and management of fetuses with liver hemangiomata. Ultrasound Obstet Gynecol. 2002;19(5):454-60. https://dx/doi.org/10.1046/ j.1469-0705.2002.00689.x. 